\title{
Response of laminated arches to impact loads
}

\author{
P. P. Procházka \& A. E. Yiakoumi \\ Czech Technical University in Prague, Civil Engineering, \\ Structural Mechanics, Czech Republic
}

\begin{abstract}
In various structures arches of a circular shape are exposed to impact loading. Such structures involve the linings of tunnels of different kinds, cockpits of aircrafts, parts of submersibles, etc. The problem is solved as pseudo three-dimensional, i.e. generalized plane strain is considered after introducing the development of time and hoop direction to the Fourier series. This assumption is in very good compliance with the natural behavior of the above mentioned structures and moreover, it enables us to describe mathematically several phenomena such as dissipation layers inside the arches, optimal distribution of reinforcement, and so on. In the radial direction the linear finite element like approach is introduced. The reason for this is the fact that the layers are considered thin enough and the explicit solution leads us to Bessel functions, which are not transparent enough. The base functions in the hoop direction are selected for simply supported arches, but the generalization of the prescribed supports is possible using the application of auspicious moments (rotations) at the end lines.
\end{abstract}

Keywords: circular laminated arches, semianalytical method, dumper layer, impact load.

\section{Introduction}

In this paper the vibration of laminated cylindrical arches subject to excitation loading is solved in the generalized plane strain state. The application can be seen in a wide scale of applications, such as in the fields of the assessment of elements of submersibles, airplanes, underground structures and others.

First the Hamiltonian is formulated in the cylindrical coordinates and the equations of equilibrium are then derived. By introducing series in the hoop 
direction and developing harmonic sine approximate loading in time and coming back to the energy formulation using test functions yields the Hamiltonian formulation for finite elements in the remaining coordinates (radial and axial directions).

Similar problem are solved in plenty of papers. To shorten the list we name only some. The vibration analysis of cylindrical shells using the wave propagation method is presented in [1]. An exact solution of free harmonic wave propagation in a composite laminated cylindrical shell is presented in [2], within the framework of the theory of three-dimensional cylindrically anisotropic elasticity. The paper [3] deals with the radial vibration of a row of cylindrical panels of infinite length using the concept of wave propagation in periodic structures.

Based on the Flugge thin shell theory, the paper [4] presents exact solutions for the vibration of circular cylindrical shells with step-wise thickness variations in the axial direction. A new method for calculating the free vibration frequencies of a thin circular cylindrical shell are presented in [5], based on Flugge's shell theory equations for orthotropic materials.

\section{Hamilton's principle}

Let us consider a laminated arch, which in an undeformed state is described by the volume $\Omega$ with boundary $\Gamma$ being equipped with the cylindrical coordinate system $0 r \theta z$, see Fig. 1. The Hamiltonian variational principle applied to the laminated arch starts with formulation of the following potential energy functional:

$$
\begin{aligned}
& \Pi=\frac{1}{2} \int_{t=0}^{T} \iiint_{\Omega} r\left(\sigma_{r} \varepsilon_{r}+\sigma_{\theta} \varepsilon_{\theta}+\sigma_{z} \varepsilon_{z}+\sigma_{r \theta} \varepsilon_{r \theta}+\sigma_{r z} \varepsilon_{r z}+\sigma_{\theta z} \varepsilon_{\theta z}\right) \mathrm{d} \Omega- \\
& -\int_{t=0}^{T} \iiint_{\Omega} r\left(X_{r} u_{r}+X_{\theta} u_{\theta}+X_{z} u_{z}\right) \mathrm{d} \Omega+ \\
& +\int_{t=0}^{T} \iiint_{\Omega} \rho r\left[\left(\frac{\partial u_{r}}{\partial t}\right)^{2}+\left(\frac{\partial u_{\theta}}{\partial t}\right)^{2}+\left(\frac{\partial u_{z}}{\partial t}\right)^{2}\right] \mathrm{d} \Omega-\int_{t=0}^{T} \iint_{\Gamma} p_{r} u_{r} \mathrm{~d} \Gamma
\end{aligned}
$$

where transformed components to the cylindrical system provide denotation of stresses as $\sigma_{r}, \sigma_{\theta}, \sigma_{z}, \sigma_{r \theta}, \sigma_{r z}, \sigma_{\theta z}$, components of the transformed strain tensor are denoted as $\varepsilon_{r}, \varepsilon_{\theta}, \varepsilon_{z}, \varepsilon_{r \theta}, \varepsilon_{r z}, \varepsilon_{\theta z}$, and $u_{r}, u_{\theta}, u_{z}$ are the displacements in the curvilinear coordinates and the external work of tractions $p_{r}$ in the radial direction are considered for the purpose of our problem, $\rho$ is the mass density, $t$ is the time. The quantities $X_{r}, X_{\theta}, X_{z}$ are components of the volume weight forces. 
Hamilton's principle states that the Hamiltonian (1) is minimum, i.e., the first variation of (1) is equal to zero. The variations of free variables, i.e. displacements in the basic three directions are known as test functions. Using Green's theorem, and considering the test functions to be arbitrary, the equations of equilibrium are formulated as:

$$
\begin{gathered}
\frac{\partial\left(r \sigma_{r}\right)}{\partial r}+\frac{\partial \sigma_{r \theta}}{\partial \theta}+\frac{\partial\left(r \sigma_{r z}\right)}{\partial z}-\sigma_{\theta}+r X_{r}=\rho r \frac{\partial^{2} u_{r}}{\partial t^{2}} \\
\frac{\partial \sigma_{\theta}}{\partial \theta}+\frac{1}{r}\left[\frac{\partial\left(r^{2} \sigma_{\theta z}\right)}{\partial z}+\frac{\partial\left(r^{2} \sigma_{r \theta}\right)}{\partial r}\right]+r X_{\theta}=\rho r \frac{\partial^{2} u_{\theta}}{\partial t^{2}} \\
\frac{\partial\left(r \sigma_{z}\right)}{\partial z}+\frac{\partial\left(r \sigma_{r z}\right)}{\partial r}+\frac{\partial \sigma_{\theta z}}{\partial \theta}+r X_{z}=\rho r \frac{\partial^{2} u_{z}}{\partial t^{2}}
\end{gathered}
$$

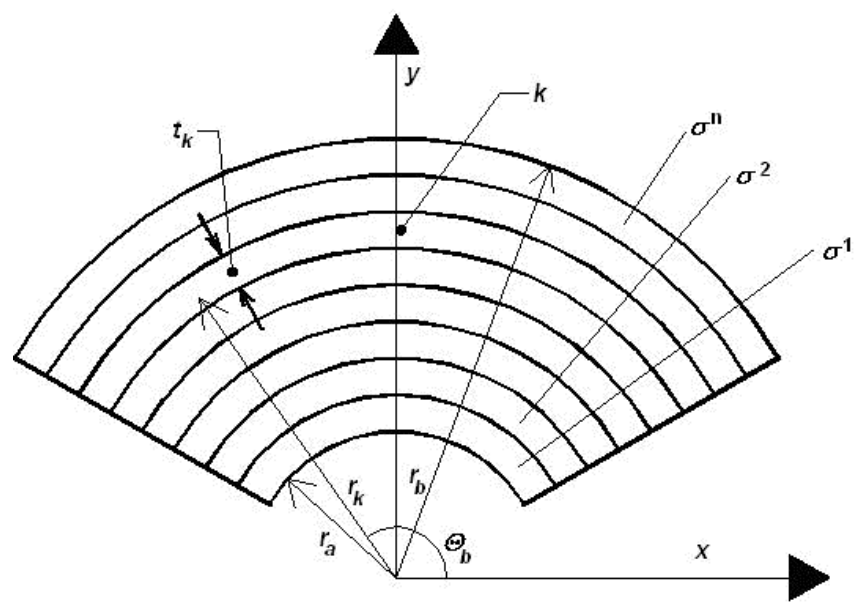

Figure 1: Geometry and denotation of quantities in laminas of an arch.

Assuming the solution in the form:

$$
\begin{aligned}
& u_{r}(r, \theta, z, t)=\sum_{i=1}^{M} \sum_{j=1}^{\Theta} u_{r}^{i j}(r, z) \sin \left(\alpha_{i} \theta\right) \sin (j \omega t) \\
& u_{\theta}(r, \theta, z, t)=\sum_{i=1}^{M} \sum_{j=1}^{\Theta} u_{\theta}^{i j}(r, z) \cos \left(\alpha_{i} \theta\right) \sin (j \omega t) \\
& u_{z}(r, \theta, z, t)=\sum_{i=1}^{M} \sum_{j=1}^{\Theta} u_{z}^{i j}(r, z) \sin \left(\alpha_{i} \theta\right) \sin (j \omega t)
\end{aligned}
$$


We express $\alpha_{i}$ as $\alpha_{i}=i . m$, where positive integer $i$ is the number of a wave in the Fourier series in the circumferential (hoop) direction and $m=\frac{\pi}{\beta}$. Angle $\beta$ identifies the end points of the arch. The kinematical equations become:

$$
\begin{gathered}
\varepsilon_{r}=\frac{\partial u_{r}}{\partial r}=\sum_{i=1}^{M} \sum_{j=1}^{\Theta} \frac{\partial u_{r}^{i j}}{\partial r} \sin \left(\alpha_{i} \theta\right) \sin (j \omega t), \\
\varepsilon_{z}=\frac{\partial u_{z}}{\partial z}=\sum_{i=1}^{M} \sum_{j=1}^{\Theta} \frac{\partial u_{z}^{i j}}{\partial z} \sin \left(\alpha_{i} \theta\right) \sin (j \omega t), \\
\varepsilon_{\theta}=\frac{1}{r} \frac{\partial u_{\theta}}{\partial \theta}+\frac{u_{r}}{r}=\sum_{i=1}^{M} \sum_{j=1}^{\Theta} \frac{\left(u_{r}^{i j}-\alpha_{i} u_{\theta}^{i j}\right)}{r} \sin \left(\alpha_{i} \theta\right) \sin (j \omega t), \\
\varepsilon_{r \theta} \frac{1}{r} \frac{\partial u_{r}}{\partial \theta}+\frac{\partial u_{\theta}}{\partial r}-\frac{u_{\theta}}{r}=\sum_{i=1}^{M} \sum_{j=1}^{\Theta}\left[\frac{\alpha_{i} u_{r}^{i j}-u_{\theta}^{i j}}{r}+\frac{\partial u_{\theta}^{i j}}{\partial r}\right] \cos \left(\alpha_{i} \theta\right) \sin (j \omega t) \\
\varepsilon_{r z}=\frac{\partial u_{z}}{\partial r}+\frac{\partial u_{r}}{\partial z}=\sum_{i=1}^{M} \sum_{j=1}^{\Theta}\left[\frac{\partial u_{z}^{i j}}{\partial r}+\frac{\partial u_{r}^{i j}}{\partial z}\right] \sin \left(\alpha_{i} \theta\right) \sin (j \omega t) \\
\varepsilon_{\theta z}=\frac{\partial u_{\theta}}{\partial z}+\frac{1}{r} \frac{\partial u_{z}}{\partial \theta}=\sum_{i=1}^{M} \sum_{j=1}^{\Theta}\left[\frac{\partial u_{\theta}^{i j}}{\partial z}+\frac{\alpha_{i} u_{z}^{i j}}{r}\right] \cos \left(\alpha_{i} \theta\right) \sin (j \omega t)
\end{gathered}
$$

Hence, from Hooke's law one gets:

$$
\begin{gathered}
\sigma_{r}=L_{r r} \varepsilon_{r}+L_{r \theta} \varepsilon_{\theta}+L_{r z} \varepsilon_{z}=\sum_{i=1}^{M} \sum_{j=1}^{\Theta} \sigma_{r}^{i j}= \\
=\sum_{i=1}^{M} \sum_{j=1}^{\Theta}\left[L_{r r} \frac{\partial u_{r}^{i j}}{\partial r}+L_{r \theta} \frac{\left(u_{r}^{i j}-\alpha_{i} u_{\theta}^{i j}\right)}{r}+L_{r z} \frac{\partial u_{z}^{i j}}{\partial z}\right] \sin \left(\alpha_{i} \theta\right) \sin (j \omega t) \\
\sigma_{\theta}=L_{r \theta} \varepsilon_{r}+L_{\theta \theta} \varepsilon_{\theta}+L_{\theta z} \varepsilon_{z}=\sum_{i=1}^{M} \sum_{j=1}^{\Theta} \sigma_{\theta}^{i j}= \\
=\sum_{i=1}^{M} \sum_{j=1}^{\Theta}\left[L_{r \theta} \frac{\partial u_{r}^{i j}}{\partial r}+L_{\theta \theta} \frac{\left(u_{r}^{i j}-\alpha_{i} u_{\theta}^{i j}\right)}{r}+L_{\theta z} \frac{\partial u_{z}^{i j}}{\partial z}\right] \sin \left(\alpha_{i} \theta\right) \sin (j \omega t) \\
\sigma_{z}=L_{r z} \varepsilon_{r}+L_{\theta z} \varepsilon_{\theta}+L_{z z} \varepsilon_{z}=\sum_{i=1}^{M} \sum_{j=1}^{\Theta} \sigma_{z}^{i j}=\sum_{i=1}^{M} \sum_{j=1}^{\Theta}\left[L_{r z} \frac{\partial u_{r}^{i j}}{\partial r}+L_{\theta z} \frac{\left(u_{r}^{i j}-\alpha_{i} u_{\theta}^{i j}\right)}{r}+L_{z z} \frac{\partial u_{z}^{i j}}{\partial z}\right] \sin \left(\alpha_{i} \theta\right) \sin (j \omega t) \\
\sigma_{r \theta}=G_{r \theta} \varepsilon_{r \theta} \\
=\sum_{i=1}^{M} \sum_{j=1}^{\Theta} \sigma_{r \theta}^{i j}=\sum_{i=1}^{M} \sum_{j=1}^{\Theta} G_{r \theta}\left[\frac{\alpha_{i} u_{r}^{i j}-u_{\theta}^{i j}}{r}+\frac{\partial u_{\theta}^{i j}}{\partial r}\right] \cos \left(\alpha_{i} \theta\right) \sin (j \omega t)
\end{gathered}
$$




$$
\begin{gathered}
\sigma_{r z}=G_{r z} \varepsilon_{r z}=\sum_{i=1}^{M} \sum_{j=1}^{\Theta} \sigma_{r z}^{i j}=\sum_{i=1}^{M} \sum_{j=1}^{\Theta} G_{r z}\left[\frac{\partial u_{z}^{i j}}{\partial r}+\frac{\partial u_{r}^{i j}}{\partial z}\right] \sin \left(\alpha_{i} \theta\right) \sin (j \omega t) \\
\sigma_{\theta z}=G_{\theta z} \varepsilon_{\theta z}=\sum_{i=1}^{M} \sum_{j=1}^{\Theta} \sigma_{\theta z}^{i j}=\sum_{i=1}^{M} \sum_{j=1}^{\Theta} G_{\theta z}\left[\frac{\partial u_{\theta}^{i j}}{\partial z}+\frac{\alpha_{i} u_{z}^{i j}}{r}\right] \cos \left(\alpha_{i} \theta\right) \sin (j \omega t)
\end{gathered}
$$

Because the displacements have developed in the hoop direction we get:

$$
\begin{gathered}
\frac{\partial \sigma_{\theta}}{\partial \theta}=\sum_{i=1}^{M} \sum_{j=1}^{\Theta} \alpha_{i}\left[L_{r \theta} \frac{\partial u_{r}^{i j}}{\partial r}+L_{\theta \theta} \frac{\left(u_{r}^{i j}-\alpha_{i} u_{\theta}^{i j}\right)}{r}+L_{\theta z} \frac{\partial u_{z}^{i j}}{\partial z}\right] \cos \left(\alpha_{i} \theta\right) \sin (j \omega t) \\
\frac{\partial \sigma_{r \theta}}{\partial \theta}=-\sum_{i=1}^{M} \sum_{j=1}^{\Theta} G_{r \theta} \alpha_{i}\left[\frac{\left(\alpha_{i} u_{r}^{i j}-u_{\theta}^{i j}\right)}{r}+\frac{\partial u_{\theta}^{i j}}{\partial r}\right] \sin \left(\alpha_{i} \theta\right) \sin (j \omega t) \\
\frac{\partial \sigma_{\theta z}}{\partial \theta}=-\sum_{i=1}^{M} \sum_{j=1}^{\Theta} G_{\theta z} \alpha_{i}\left[\frac{\partial u_{\theta}^{i j}}{\partial z}+\frac{\alpha_{i} u_{z}^{i j}}{r}\right] \sin \left(\alpha_{i} \theta\right) \sin (j \omega t) \\
\frac{\partial\left(r \sigma_{r z}\right)}{\partial z}=\sum_{i=1}^{M} \sum_{j=1}^{\Theta} r G_{r z}\left[\frac{\partial^{2} u_{z}^{i j}}{\partial r \partial z}+\frac{\partial^{2} u_{r}^{i j}}{\partial z^{2}}\right] \sin \left(\alpha_{i} \theta\right) \sin (j \omega t) \\
\frac{1}{r} \frac{\partial\left(r^{2} \sigma_{\theta z}\right)}{\partial z}=\sum_{i=1}^{M} \sum_{j=1}^{\Theta} r G_{\theta z}\left[\frac{\partial^{2} u_{\theta}^{i j}}{\partial z^{2}}+\frac{\alpha_{i} \partial u_{z}^{i j}}{r \partial z}\right] \cos \left(\alpha_{i} \theta\right) \sin (j \omega t)
\end{gathered}
$$

In the following text we fully utilize the above relations in formulation of the problem in one lamina for fixed $i$ and $j$. Because of these assumptions we drop out the indices in the expressions to simplify the formulas.

\section{Solution in one lamina with fixed time and hoop waves}

Let $\Omega_{k}$ be the cross section of lamina $k$ (which is the index between 1 and $N$ being the number of laminas) with a boundary (outer, inner, or interfacial) $\Gamma_{k}$. Equations (5) and (6) can now be substituted to (2). Comparing both sides of the resulting equations, and neglecting the volume weight, leads us formally to the same equations as (2) which now are valid in $\Omega_{k}$.

Multiplying the first equation (2) by $\varphi_{a}$ and integrating the result over the area of the layer $k$, then again multiplying the first equation by $\varphi_{b}$ and integrating in the same way yields:

$$
\int_{\Omega_{k}} \varphi_{l}\left(\frac{\partial\left(r \sigma_{r}\right)}{\partial r}+\frac{\partial \sigma_{r \theta}}{\partial \theta}+\frac{\partial\left(r \sigma_{r z}\right)}{\partial z}-\sigma_{\theta}\right) \mathrm{d} \Omega_{k}=-(j \omega)^{2} \int_{\Omega_{k}} \varphi_{l} \rho r u_{r} \mathrm{~d} \Omega_{k}, l=a, b
$$

Applying Green's theorem to the first term of the left hand side of (9) gives: 


$$
\begin{aligned}
& \int_{\Omega_{k}}\left(-\frac{\partial \varphi_{l}}{\partial r} r \sigma_{r}+\varphi_{l} \frac{\partial \sigma_{r \theta}}{\partial \theta}+\varphi_{l} \frac{\partial\left(r \sigma_{r z}\right)}{\partial z}-\varphi_{l} \sigma_{\theta}\right) \mathrm{d} \Omega_{k}+ \\
& \quad+\int_{\Gamma_{k}} r \varphi_{l} \sigma_{r} n_{r} \mathrm{~d} \Gamma_{k}=-(j \omega)^{2} \int_{\Omega_{k}} \varphi_{l} \rho r u_{r} \mathrm{~d} \Omega_{k}, l=a, b, \mathrm{~d} \Omega_{k} \equiv \mathrm{d} r \mathrm{~d} z
\end{aligned}
$$

From this relation boundary conditions follow on $\Gamma_{k}$, which have to be precised to also involve the waves

$$
\sum_{i=1}^{M} \sum_{j=1}^{\Theta} \int_{\Gamma_{k}} r \varphi_{l} \sigma_{r}^{i j} n_{r} \mathrm{~d} \Gamma_{k} \sin \left(\alpha_{i} \theta\right) \sin (j \omega t)=\sum_{i=1}^{M} \sum_{j=1}^{\Theta} \int_{\Gamma_{k}} r \varphi_{l} p_{r}^{i j} \mathrm{~d} \Gamma_{k} \sin \left(\alpha_{i} \theta\right) \sin (j \omega t),
$$

where $p_{r}^{i j}$ are amplitudes of tractions in the radial direction on $\Gamma_{k}$. Moreover, the outward unit normal is defined by its components $\boldsymbol{n}=\left\{n_{r}, n_{\theta}, n_{z}\right\}$. In the next step we introduce approximations in the sense of general plane strain ( $\varepsilon_{z}^{i j}=$ const. for each $i$ and $j$ ):

$$
\begin{array}{ll}
u_{r}=\frac{b-r}{h} u_{r}^{a}+\frac{r-a}{h} u_{r}^{b}, \quad u_{\theta}=\frac{b-r}{h} u_{\theta}^{a}+\frac{r-a}{h} u_{\theta}^{b} \quad, \quad u_{z}=\varepsilon_{z} z \\
\varphi_{a}=\frac{b-r}{h}, \quad \varphi_{b}=\frac{r-a}{h}, \quad h=b-a
\end{array}
$$

where $r \in(a, b), a$ and $b$ are respectively inner and outer radii of the lamina $k, h$ is the thickness of the lamina, $u_{r}^{a}, u_{r}^{b}$ are the inner and outer radial displacements, $u_{\theta}^{a}, u_{\theta}^{b}$ are inner and outer displacements in the hoop direction and $\varphi_{a}, \varphi_{b}$ are the test functions. The displacements $u_{r}^{a}, u_{r}^{b} u_{\theta}^{a}, u_{\theta}^{b}$ and $\varepsilon_{z}$ are the free parameters to be solved for each $i$ and $j$. Note that in the case of one lamina and one wave the kinematical equations (4) simplify as:

$$
\varepsilon_{r}=\frac{\partial u_{r}}{\partial r}, \varepsilon_{\theta}=\frac{\left(u_{r}-\alpha u_{\theta}\right)}{r}, \varepsilon_{r \theta}=\frac{\alpha u_{r}-u_{\theta}}{r}+\frac{\partial u_{\theta}}{\partial r}, \varepsilon_{r z}=0, \varepsilon_{\theta z}=\frac{\alpha \varepsilon_{z} z}{r}
$$

Applying a similar approach to the second and the third equations (2) delivers boundary conditions, which are for completeness written again in terms involving the time and hoop waves:

$$
\sum_{i=1}^{M} \sum_{j=1}^{\Theta} \int_{\Gamma_{k}} r \varphi_{l} \sigma_{r \theta}^{i j} n_{r} \mathrm{~d} \Gamma_{k} \cos \left(\alpha_{i} \theta\right) \sin (j \omega t)=\sum_{i=1}^{M} \sum_{j=1}^{\Theta} \int_{\Gamma_{k}} r \varphi_{l} p_{\theta}^{i j} \mathrm{~d} \Gamma_{k} \cos \left(\alpha_{i} \theta\right) \sin (j \omega t),
$$


$\sum_{i=1}^{M} \sum_{j=1}^{\Theta} \int_{\Gamma_{k}} r \varphi_{z} \sigma_{z}^{i j} n_{z} \mathrm{~d} \Gamma_{k} \sin \left(\alpha_{i} \theta\right) \sin (j \omega t)=\sum_{i=1}^{M} \sum_{j=1}^{\Theta} \int_{\Gamma_{k}} r \varphi_{z} p_{z}^{i j} \mathrm{~d} \Gamma_{k} \sin \left(\alpha_{i} \theta\right) \sin (j \omega t)$,

$p_{\theta}^{i j}, p_{z}^{i j}$ are respectively amplitudes of tractions in the hoop and axial directions, $\varphi_{z}=\frac{z}{L}$ is the fifth base function being connected with the axial displacement, and $L$ is the length of the arch.

The above approach produces a variational formulation for the second equation:

$$
\begin{aligned}
\int_{\Omega_{k}}\left(\varphi_{l} \frac{\partial \sigma_{\theta}}{\partial \theta}+\varphi_{l} \frac{\partial\left(r \sigma_{\theta z}\right)}{\partial z}\right. & \left.+\varphi_{l} \sigma_{r \theta}-\frac{\partial \varphi_{l}}{\partial r} r \sigma_{r \theta}\right) \mathrm{d} \Omega_{k}+ \\
& +\int_{\Gamma_{k}} r \varphi_{l} p_{\theta} \mathrm{d} \Gamma_{k}=-(j \omega)^{2} \int_{\Omega_{k}} \varphi_{l} \rho r u_{\theta} \mathrm{d} \Omega_{k}
\end{aligned}
$$

The third equation will be multiplied by $\varphi_{z}$, integrated over $\Omega_{k}$ and Green's theorem will be applied to the first term of the left hand side to get:

$$
\begin{aligned}
\int_{\Omega_{k}}\left(-\frac{\partial \varphi_{z}}{\partial z} r \sigma_{z}+\varphi_{z} \frac{\partial\left(r \sigma_{r z}\right)}{\partial r}+\varphi_{z} \frac{\partial \sigma_{\theta z}}{\partial \theta}\right) \mathrm{d} \Omega_{k}+ \\
\quad+\int_{\Gamma_{k}} r \varphi_{z} p_{z} \mathrm{~d} \Gamma_{k}=-(j \omega)^{2} \int_{\Omega_{k}} \varphi_{z} \rho r u_{z} \mathrm{~d} \Omega_{k}
\end{aligned}
$$

In the sense of our approximation the second and the third terms of the left hand side disappear, which results in easy relation in $\Omega_{k}$ :

$$
\int_{\Omega_{k}}-\frac{\partial \varphi_{z}}{\partial z} r \sigma_{z} \mathrm{~d} \Omega_{k}+\int_{\Gamma_{k}} r \varphi_{z} p_{z} \mathrm{~d} \Gamma_{k}=-(j \omega)^{2} \int_{\Omega_{k}} \varphi_{z} \rho r u_{z} \mathrm{~d} \Omega_{k}
$$

The average value of the stress components over the thickness of the layer are defined as:

$$
<\sigma_{z z}>=\frac{2}{b^{2}-a^{2}} \int_{a}^{b} r \sigma_{z z} \mathrm{~d} r
$$

Moreover, we define the boundaries of the layer:

$$
P_{a}=-\left(p_{a} a\right), P_{b}=p_{b} b, Q_{a}=-\left(q_{a} a\right), Q_{b}=q_{b} b, P_{z}=<\sigma_{z z}>\frac{b^{2}-a^{2}}{2}
$$

where $p_{a}=\sigma_{r r}, q_{a}=\sigma_{r \theta} @ r=a, p_{b}=\sigma_{r r}, q_{b}=\sigma_{r \theta} @ r=b$ and we write the force-displacement relation for the single layer under the boundary conditions as:

$$
\left\{\begin{array}{lllll}
P_{a} & Q_{a} & P_{b} & Q_{b} & P_{z}
\end{array}\right\}^{\mathrm{T}}=\boldsymbol{K}-(j \omega)^{2} \boldsymbol{M}\left\{\begin{array}{lllll}
u_{r}^{a} & u_{\theta}^{a} & u_{r}^{b} & u_{\theta}^{b} & u_{z}
\end{array}\right\}^{\mathrm{T}}
$$

where the coefficients of $\boldsymbol{K}$ are evaluated from (10), (16) and (18) and $\boldsymbol{M}$ is the mass matrix. 


\section{Entire structure}

In order to get relations in the entire structure, interfacial and boundary conditions have to be applied. The boundary conditions have to obey either prescribed displacements in the radial, magnitudes in hoop or/and axial directions. The other possibility is to prescribe tractions $\boldsymbol{p}$, which are connected with stresses according to such formulas:

$$
\begin{gathered}
p_{a}=\sigma_{r r}, q_{a}=\sigma_{r \theta} @ \text { inner boundary and } p_{b}=\sigma_{r r}, q_{b}=\sigma_{r \theta} @ \text { outer boundary } \\
p_{z}=P_{z} @ \text { the face } z=L
\end{gathered}
$$

On the interfaces between layers we apply interfacial conditions of the following kind: the displacements are continuous and the tractions are in equilibrium and the relations (21) are used.

Generally we get $N \times M$ systems of equations for each wave $i=1, \ldots, N$ in the hoop direction times each wave $j=1, \ldots, M$ in the time scale. For eigenvalues of frequencies (natural frequencies) put $j=1$ and select successively $i=1, \ldots, N$. Eventually we arrive at the overall system of equations for solving either eigenfrequencies or reaction on the external excitation, which has to be developed into sine serious in time. This can formally be recorded as:

$$
\left[\left(\boldsymbol{K}^{1} \oplus \boldsymbol{K}^{2} \oplus \ldots \oplus \boldsymbol{K}^{N}\right)-(j \omega)^{2}\left(\boldsymbol{M}^{1} \oplus \boldsymbol{M}^{2} \oplus \ldots \oplus \boldsymbol{M}^{N}\right)\right]\{\boldsymbol{u}\}=\{\boldsymbol{p}\}
$$

where $\oplus$ is a symbol for generalized addition in the sense of finite elements, $\{\boldsymbol{u}\}$ is the vector of displacements and tractions $\{\boldsymbol{p}\}$ in $r, \theta, z$ directions.

\section{Example}

In order to suppress an impact of explosive load on the outer boundary (the inner boundary is free of load) a dumper is positioned in various layers. In connection with the position inside the laminated arch, different natural frequencies are attained. In this way the vector $\{\boldsymbol{p}\}$ in (22) disappears, $j=1$, or $j=2$ and the eigenvalue problems are to be solved from (22).

The arch has the following dimensions and material properties: $l=1 \mathrm{~m}, r=2$ $\mathrm{m}, t=5 \mathrm{~mm}, \beta=0.5 \mathrm{rad}, E=208 \times 10^{9} \mathrm{~N} / \mathrm{m}^{2}, \rho=7833 \mathrm{~kg} / \mathrm{m}^{3}, v=0.29$.

Five equidistant layers are considered through the thickness of the arch. The dumper positioned in only one layer with $E=200 \times 10^{8} \mathrm{~N} / \mathrm{m}^{2}$ will move for successive examples from the layer one to five. The natural frequencies are calculated for these particular cases. They are displayed in Fig. 2. Number one belongs to the position of the dumper in layer one, etc., the numbering is from the outer boundary to the lower one.

\section{Conclusions}

In this paper the procedure for calculating a laminated arch is suggested in cylindrical coordinates $0 r \theta z$, starting with the assumption of generalized plain 


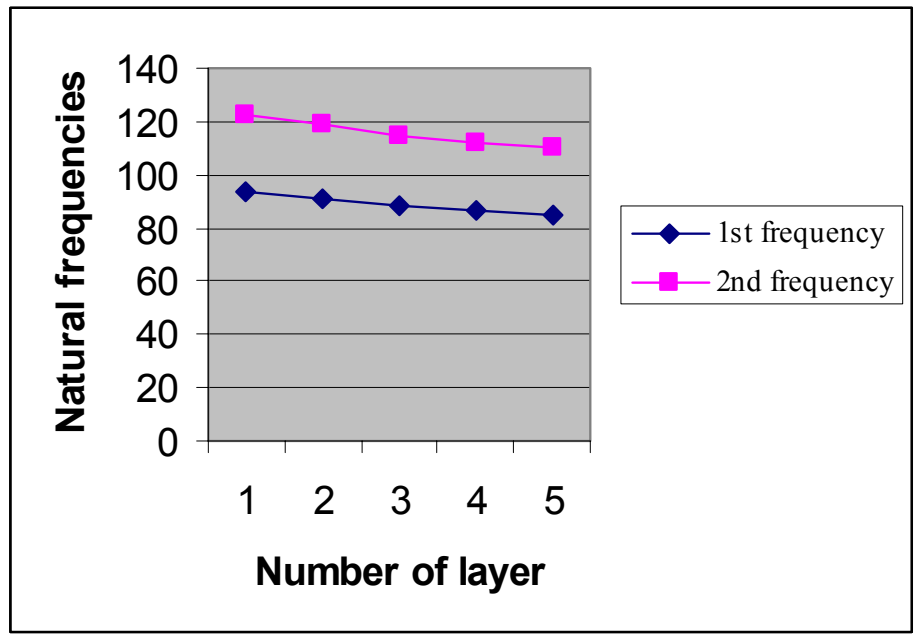

Figure 2: Natural frequencies for various positions of the dumper.

strain. Before introducing this assumption, the displacements are developed into Fourier's series in the time and hoop coordinates. Employing kinematical equations in cylindrical coordinates and Hooke's law, the stresses are derived in the split formulation, from which the radial and axial coordinates on one side and the time and hoop coordinates on the other side are separated. After this, variational formulation follows and a finite element-like procedure is employed in the coordinate system $0 \mathrm{rz}$. In the radial direction linear approximation of displacements is supposed and in the sense of the generalized plane strain linear distribution of displacements in the axial direction is also introduced. A simply supported segment is considered in our case, but more general supports can be involved using given moments at the end points, the clamped edge can be simulated, for example.

As an example of the application of the above described approach a dumping layer for the dissipation of energy after the application of an explosive load is considered in various laminates. Two first natural (eigen) frequencies are observed dependent on the position in the structure of the arch. It appears that the most promising case is that which is defined by positioning the dumper towards the outer boundary.

\section{Acknowledgement}

This paper was prepared under financial support of GAČR, project No. 103/08/0922. 


\section{References}

[1] Zhang, X.M., Liu, G.R., and Lam K.Y. Vibration analysis of thin cylindrical shells using wave propagation approach. Journal of Sound and Vibration 239, 3, 2001, 397-403

[2] Yuan, F.G., Hsieh, C.C. Three-dimensional wave propagation in composite cylindrical shells. Composite Structures 42, 2,1998,153-167

[3] Pany C., Parthan, S., Mukherjee, S. Vibration analysis of multi-supported curved panel using the periodic structure approach. International Journal of Mechanical Sciences 44, 2, 2002, 269-285

[4] Zhang, L., Xiang Y. Exact solutions for vibration of stepped circular cylindrical shells. Journal of Sound and Vibration 299, 4-5, 2007, 948-964

[5] Xuebin, L. A new approach for free vibration analysis of thin circular cylindrical shell. Journal of sound and vibration 296,1-2, 2006, 91-98 